Pacific Journal of Mathematics

GENERALIZED COMPLETE MAPPINGS, NEOFIELDS,
SEQUENCEABLE GROUPS AND BLOCK DESIGNS. II 


\title{
GENERALIZED COMPLETE MAPPINGS, NEOFIELDS, SEQUENCEABLE GROUPS AND BLOCK DESIGNS. II
}

\author{
D. F. Hsu AND A. D. KeEDwell
}

\begin{abstract}
In part I we introduced the concepts of generalized complete mapping and generalized near complete mapping of a group and used them to characterize left neofields. In $\$ 5$ of this second part we describe how $(K, \lambda)$ complete mappings and near complete mappings may be used to provide new constructions of block designs of Mendelsohn type; and in $\S 6$ we describe various methods of obtaining generalized complete mappings.
\end{abstract}

For the convenience of the reader we again give the definitions of these concepts, but for all other matters we refer to part I. We denote the sections of this paper by 5 and 6 (those of the earlier paper being 1 to 4 ) and use decimal notation for the numbering of theorems and definitions. A reference to Theorem 2.1 is therefore a reference to the first theorem in $\$ 2$ of part I. However, bibliographical references for this second part are independent of those in part $I$.

A number of examples illustrative of the various concepts which we introduce will be found in the Appendix. We shall refer to them by number.

Definitions of a $(K, \lambda)$ complete mapping and $(K, \lambda)$ near complete mapping (Definition 2.4 of part I).

$A(K, \lambda)$ complete mapping, where $K=\left\{k_{1}, k_{2}, \ldots, k_{s}\right\}$ and the $k_{i}$ are integers such that $\sum_{i=1}^{s} k_{i}=\lambda(|G|-1)$, is an arrangement of the non-identity elements of a group $G$ (each used $\lambda$ times) into $s$ cyclic sequences (of distinct elements) of lengths $k_{1}, k_{2}, \ldots, k_{s}$, say

$$
\left(g_{11} g_{12} \cdots g_{1 k_{1}}\right)\left(g_{21} g_{22} \cdots g_{2 k_{2}}\right) \cdots\left(g_{s 1} g_{s 2} \cdots g_{s k_{s}}\right) \text {, }
$$

such that the elements $g_{i j}^{-1} g_{i, j+1}$ (where $i=1,2, \ldots, s$; and the second suffix $j$ is added modulo $k_{i}$ ) comprise the non-identity elements of $G$ each counted $\lambda$ times.

$A(k, \lambda)$ near complete mapping, where $K=\left\{h_{1}, h_{2}, \ldots, h_{r}\right.$; $\left.k_{1}, k_{2}, \ldots, k_{s}\right\}$ and the $h_{i}$ and $k_{j}$ are integers such that $\sum_{i=1}^{r} h_{i}+\sum_{j=1}^{s} k_{j}=$ $\lambda|G|$, is an arrangement of the elements of $G$ (each used $\lambda$ times) into $r$ sequences with lengths $h_{1}, h_{2}, \ldots, h_{r}$ and $s$ cyclic sequences with lengths 
$k_{1}, k_{2}, \ldots, k_{s}$, say,

$$
\begin{array}{r}
{\left[g_{11}^{\prime} g_{12}^{\prime} \cdots g_{1 h_{1}}^{\prime}\right] \cdots\left[g_{r 1}^{\prime} g_{r 2}^{\prime} \cdots g_{r h_{r}}^{\prime}\right]\left(g_{11} g_{12} \cdots g_{1 k_{1}}\right)} \\
\cdots\left(g_{s 1} g_{s 2} \cdots g_{s k_{s}}\right) \\
\text {. }
\end{array}
$$

such that the elements $\left(g_{l j}^{\prime}\right)^{-1} g_{i, j+1}^{\prime}$ and $g_{l j}^{-1} g_{l, j+1}$ together with the elements $g_{\imath k_{t}}^{-1} g_{i 1}$ comprise the non-identity elements of $G$ each counted $\lambda$ times. (We have $\sum\left(h_{l}-1\right)+\sum k_{j}=\lambda(|G|-1)$ so it is immediate from the definition itself that $r=\lambda$.)

Definitions of $a(k, \lambda)$ complete mapping and $a(k, \lambda)$ near complete mapping (Definition 2.5 of part I)

$A(k, \lambda)$ complete mapping is a $(K, \lambda)$ complete mapping such that $K=\{k, k, \ldots, k\}$. For such a generalized complete mapping, $s=$ $\lambda(|G|-1) / k$.

Similarly, $a(k, \lambda)$ near complete mapping is a $(K, \lambda)$ near complete mapping such that $K=\{h, h, \ldots, h ; k, k, \ldots, k\}$ and $k-h=1$.

5. Block designs of Mendelsohn type. In this section we show how the concepts of $(K, \lambda)$ complete mapping and $(K, \lambda)$ near complete mapping can be used to construct block designs of Mendelsohn type whose automorphism group contains a specified group $G$ as subgroup. For this purpose it is convenient to use additive notation for cyclic groups.

Definition 5.1. A block design of Mendelsohn type comprises a set $G$ of $v$ elements and a collection of $b$ cyclically ordered subsets of $G$ called blocks of cardinalities $k_{1}, k_{2}, \ldots, k_{b}$, respectively, with the property that every ordered pair of elements of $G$ are consecutive in exactly $\lambda$ of the blocks. We call such a design a $(v, K, \lambda)$ Mendelsohn design, where $K$ is the set formed by the distinct integers among $k_{1}, k_{2}, \ldots, k_{b}$. More briefly, we shall write it as a $(v, K, \lambda)$-MD and denote the set of blocks by $B$. (See Examples 1-3.)

Definition 5.2. A $(v, k, \lambda)$-MD is a $(v, K, \lambda)$-MD such that $k_{1}=k_{2}$ $=\cdots=k_{b}=k$. (See Examples 2-5.)

In Example 2 of the Appendix the mapping $\alpha: i \rightarrow i+1, \infty \rightarrow \infty$ maps $B_{i j}$ onto $B_{i+1, j}$, where $B=\bigcup_{i=0}^{12}\left\{B_{i j}: j=1,2,3,4\right\}$, and defines an automorphism of the Mendelsohn design $(G, B)$, which permutes both the objects and blocks, each in a cycle of length 13 . This example and also Example 4 suggest the following concept. 
Definition 5.3. Let $D=(G, B)$ be a $(v, K, \lambda)$-MD and let $L$ be a group of $v$ permutations $\alpha_{1}, \alpha_{2}, \ldots, \alpha_{v}$ of $G$ such that $G=\bigcup_{i=1}^{v} \alpha_{i}(g)$, where $g$ is any fixed element of $G$. Suppose further that there exists a subset $B^{*}=\left\{B_{1}, B_{2}, \ldots, B_{f}\right\}$ of blocks of $B$ such that $B=$ $\bigcup_{i=1}^{v}\left\{\alpha_{i}\left(B_{1}\right), \alpha_{i}\left(B_{2}\right), \ldots, \alpha_{i}\left(B_{f}\right)\right\}$. Then it follows that each permutation of $L$ permutes the blocks of $B$ among themselves and is an automorphism of $D$. We say that $D$ admits $L$ as a regular group of automorphisms with the blocks of $B^{*}$ as basis blocks.

We observe that, in Examples 2 and 4 of the Appendix, the elements of $G$ are themselves elements of a group. In each case the left regular repesentation $L_{G}$ of this group has the properties required by Definition

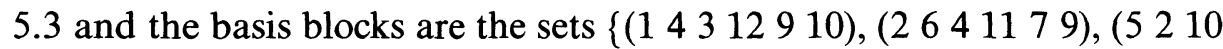
$\left.8113),\left(\begin{array}{lllll}6 & 1 & 8 & 7 & 12\end{array}\right)\right\}$ and $\left.\left\{a a^{2} b a^{2} b a b a^{3} a^{3} b\right)\right\}$, respectively. Moreover, in each case, the basis blocks define the cyclic sequences of a $(K, \lambda)$ complete mapping of the group defined on the set $G$.

We may state the following:

THEOREM 5.1. Let $L_{G}$ be the left regular representation of a group $(G, \cdot)$ of order $v$ and suppose that there exists $a(v, K, \lambda)$-MD defined on the set $G$ which admits $L_{G}$ as a regular group of automorphisms with the blocks

$$
\left(g_{11} g_{12} \cdots g_{1 k_{1}}\right),\left(g_{21} g_{22} \cdots g_{2 k_{2}}\right) \cdots\left(g_{s 1} g_{s 2} \cdots g_{s k_{s}}\right)
$$

as basis blocks, where $K=\left\{k_{1}, k_{2}, \ldots, k_{s}\right\}$ and where one element $x$ of $G$ does not occur at all among the elements of the basis blocks but every other element of $G$ occurs exactly $\lambda$ times, then the cyclic sequences

$$
\begin{array}{r}
\left(x^{-1} g_{11} x^{-1} g_{12} \cdots x^{-1} g_{1 k_{1}}\right),\left(x^{-1} g_{21} x^{-1} g_{22} \cdots x^{-1} g_{2 k_{2}}\right), \ldots, \\
\left(x^{-1} g_{s 1} x^{-1} g_{s 2} \cdots x^{-1} g_{s k_{s}}\right)
\end{array}
$$

form a $(K, \lambda)$ complete mapping of the group $(G, \cdot)$.

Conversely, if

$$
\left(g_{11} g_{12} \cdots g_{1 k_{1}}\right),\left(g_{21} g_{22} \cdots g_{2 k_{2}}\right), \ldots,\left(g_{s 1} g_{s 2} \cdots g_{s k_{s}}\right)
$$

is a $(K, \lambda)$ complete mapping of a group $(G, \cdot)$ of order $v$ with $K=$ $\left\{k_{1}, k_{2}, \ldots, k_{s}\right\}$ then the blocks of the set

$$
B=\bigcup_{g \in G}\left\{\left(g g_{11} g g_{12} \cdots g g_{1 k_{1}}\right),\left(g g_{21} g g_{22} \cdots g g_{2 k_{2}}\right), \ldots,\right.
$$

$$
\left.\left(g g_{s 1} g g_{s 2} \cdots g g_{s k_{s}}\right)\right\}
$$

form $a(v, K, \lambda)-M D$ which admits the left regular representation $L_{G}$ of 
$(G, \cdot)$ as a regular group of automorphisms such that the cyclic sequences which define the $(K, \lambda)$ complete mapping of $G$ are its basis blocks.

Proof. For the first part, we wish to show that the elements $\left(x^{-1} g_{i j}\right)^{-1}$, $\left(x^{-1} g_{i, j+1}\right)$, where $i=1,2, \ldots, s$ and the second suffix $j$ is added modulo $k_{i}$, comprise the non-identity elements of $G$ each counted $\lambda$ times. We know that each of the ordered pairs $(g, g u)$, where $u$ is an assigned element of $G$ and $g$ varies through the set $G$, occurs as a pair of consecutive elements $\lambda$ times among the blocks of the set

$$
\begin{array}{r}
B=\bigcup_{y \in G}\left\{\left(y g_{11} y g_{12} \cdots y g_{1 k_{1}}\right),\left(y g_{21} y g_{22} \cdots y g_{2 k_{2}}\right), \ldots,\right. \\
\left.\left(y g_{s 1} y g_{s 2} \cdots y g_{s k_{s}}\right)\right\} .
\end{array}
$$

Suppose that the ordered pair $\left(y g_{i j}, y g_{i, j+1}\right)$ is one of these occurrences. Then $g=y g_{i j}$ and $g u=y g_{i, j+1}$. So

$$
u=\left(y g_{\imath \jmath}\right)^{-1}\left(y g_{i, j+1}\right)=g_{i j}^{-1} g_{\imath, j+1}=\left(x^{-1} g_{i j}\right)^{-1}\left(x^{-1} g_{i, j+1}\right) \text {. }
$$

No two of the ordered pairs $\left(y g_{i j}, y g_{i, j+1}\right)$ which are equal to $(g, g u)$ can involve the same $g_{i j}$ since otherwise they would also involve the same $y$. It follows that the element $u$ of $G$ appears $\lambda$ times among the elements $\left(x^{-1} g_{i j}\right)^{-1}\left(x^{-1} g_{i, j+1}\right)$, as required.

For the converse, we wish to show first that each ordered pair of elements of $G$ occurs consecutively $\lambda$ times among the blocks of the set $B$ defined in the statement of the theorem. Let $(u, v)$ be an ordered pair of elements of $G$. In the given $(K, \lambda)$ complete mapping of the group $(G, \cdot)$, the element $u^{-1} w$ occurs $\lambda$ times in the form $g_{i j}^{-1} g_{i, j+1}$ for suitable values of $i$ and $j$. Define $t$ as the solution of the equation $g g_{i j}=u$. Then $w=$ $u\left(u^{-1} w\right)=g g_{i, j+1}$ so the ordered pair $\left(g g_{i,}, g g_{i, j+1}\right)$ for this value of $g$ is the pair $(u, w)$. It follows that the pair $(u, w)$ occurs $\lambda$ times among the blocks of $B$ and so $(G, B)$ is a $(v, K, \lambda)$-MD. The remaining statement of the theorem follows immediately from Definition 5.3.

COROLlaRY. If the group $(G, \cdot)$ of order $v$ is $R$-sequenceable then there exists $a(v, v-1,1)-M D$ which admits the group $L_{G}$ of permutations of the left regular representation of $(G, \cdot)$ as a regular group of automorphisms. (See Example 6.)

THEOREM 5.2. If

$$
\begin{array}{r}
{\left[g_{11}^{\prime} g_{12}^{\prime} \cdots g_{1 h_{1}}^{\prime}\right] \cdots\left[g_{\lambda 1}^{\prime} g_{\lambda 2}^{\prime} \cdots g_{\lambda h_{\lambda}}^{\prime}\right]\left(g_{11} g_{12} \cdots g_{1 k_{1}}\right)} \\
\cdots\left(g_{s 1} g_{s 2} \cdots g_{s k_{s}}\right)
\end{array}
$$


is a $(K, \lambda)$ near complete mapping of a group $(G, \cdot)$ of order $v-1$ with $K=\left\{h_{1}, h_{2}, \ldots, h_{\lambda} ; k_{1}, k_{2}, \ldots, k_{s}\right\}$ then the blocks of the set

$$
\begin{array}{r}
B=\bigcup_{g \in G}\left\{\left(g g_{11}^{\prime} g g_{12}^{\prime} \cdots g g_{1 h_{1}}^{\prime} \infty\right),\left(g g_{21}^{\prime} g g_{22}^{\prime} \cdots g g_{2 h_{2}}^{\prime} \infty\right), \ldots,\right. \\
\left(g g_{\lambda 1}^{\prime} g g_{\lambda 2}^{\prime} \cdots g g_{\lambda h_{\lambda}}^{\prime} \infty\right),\left(g g_{11} g g_{12} \cdots g g_{1 k_{1}}\right), \\
\left.\left(g g_{21} g g_{22} \cdots g g_{2 k_{2}}\right), \ldots,\left(g g_{s 1} g g_{s 2} \cdots g g_{s k_{s}}\right)\right\}
\end{array}
$$

form $a\left(v, k^{*}, \lambda\right)-M D$ on the set $G^{*}=G \cup\{\infty\}$, where $K^{*}=\left\{h_{1}+1\right.$, $\left.h_{2}+1, \ldots, h_{\lambda}+1 ; k_{1}, k_{2}, \ldots, k_{s}\right\}$. Moreover the group of permutations

$$
\bigcup_{g \in G}\left(\begin{array}{rrrrr}
g_{1} & g_{2} & \cdots & g_{v-1} & \infty \\
g g_{1} & g g_{2} & \cdots & g g_{v-1} & \infty
\end{array}\right) \quad \text { where } G=\left\{g_{1}, g_{2}, \ldots, g_{v-1}\right\} \text {, }
$$

acts as a regular group of automorphisms on this design with basis blocks

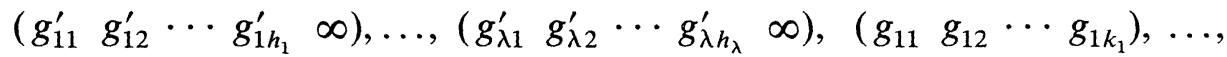
$\left(g_{s 1} g_{s 2} \cdots g_{s k_{s}}\right)$.

Proof. It is easy to check directly that each ordered pair $(g, \infty)$ and also each ordered pair $(\infty, g)$ occur $\lambda$ times as a pair of consecutive elements among the blocks of the set $B$. The remainder of the proof is exactly similar to that of the second part of Theorem 5.1.

COROLlaRY. If the group $(G, \cdot)$ of order $v-1$ is sequenceable then there exists $a(v, v, 1)-M D$ which admits the group of permutations

$$
\bigcup_{g \in G}\left(\begin{array}{ccccc}
g_{1} & g_{2} & \cdots & g_{v-1} & \infty \\
g g_{1} & g g_{2} & \cdots & g g_{v-1} & \infty
\end{array}\right)
$$

as a regular group of automorphisms.

Examples 7 and 8 illustrate Theorem 5.2.

As special cases of Theorems 5.1 and 5.2, we see that a $(k, \lambda)$ complete mapping of a group of order $v$ and a $(k, \lambda)$ near complete mapping of a group of order $v-1$ each give rise to a $(v, k, \lambda)-\mathrm{MD}$. We consider next the question of when such a design is resolvable.

Since the number of blocks in a $(v, k, \lambda)-\mathrm{MD}$ is $\lambda v(v-1) / k$, which must be an integer, we have $v(v-1) \equiv 0 \bmod k$ in the case when $\lambda=1$.

Definition 5.4. If the blocks of $(v, k, 1)$-MD for which $v \equiv 1 \bmod k$ can be partitioned into $v$ sets each containing $(v-1) / k$ blocks which are pairwise disjoint (as sets), we say that the $(v, k, 1)-\mathrm{MD}$ is resolvable and any such parititon is called a resolution of the design. 
Each set of $(v-1) / k$ pairwise disjoint blocks together with the singleton which is the only element not in any of its blocks is called a parallel class of the resolution. Any resolution of this kind has $v$ parallel classes.

\section{These definitions were first given in [2].}

DEFINITION 5.5. If the blocks of a $(v, k, 1)$-MD for which $v \equiv 0 \bmod k$ can be partitioned into $v-1$ sets each containing $v / k$ blocks which are pairwise disjoint (as sets), we shall again say that the $(v, k, 1)$-MD is resolvable.

Each set of $v / k$ pairwise disjoint blocks will be called a parallel class.

A Mendelsohn design which is resolvable in either of the above senses will be denoted as a $(v, k, 1)$-RMD.

By Theorem 5.1, Theorem 5.2 and the above two definitions, we have

THEOREM 5.3. Every $(v, k, 1)$-MD obtained from a $(k, 1)$ complete mapping in the manner of Theorem 5.1 is resolvable. Likewise, every $(v, k, 1)-\mathrm{MD}$ derived from $a(k, 1)$ near complete mapping in the manner of Theorem 5.2 is resolvable.

Note that by Theorems 5.1 and 5.3, a $(v, k, 1)$-MD derived from a $(k, 1)$ complete mapping of a cyclic group must be cyclic ${ }^{1}$ and resolvable. It is proved in [2] and [3] that a $(v, 3,1)-\mathrm{RMD}$ exists if $v \equiv 1(\bmod 3)$ and in [4] that a $(v, 3,1)-\mathrm{RMD}$ exists if $v \equiv 0(\bmod 3)$. On the other hand, it is proved in [5] that a cyclic $(v, 3,1)$-MD exists if and only if $v \equiv 1$ or 3 $(\bmod 6), v \neq 9$. It is conjectured (see [7] and $[8])$ that $(3,1)$ complete mappings of the cyclic group $C_{v}$ exist for all $v \equiv 1(\bmod 6)$. If this were proved, we should then have that cyclic $(v, 3,1)$-RMD's exist if and only if $v \equiv 1(\bmod 6)$ (the other direction of the implication is easy to see). We shall give a general account of the construction of complete mappings and near complete mappings in the next section.

Definition 5.6. Let $S$ be a given set and let $B_{l}=\left(a_{l 1} a_{l 2} \cdots a_{l k}\right)$ be a cyclically ordered subset of $k$ elements of $S$. Then the elements $a_{i r}$ and $a_{i, r+t}$, where addition of the second suffix is modulo $k$, are said to be $t$-apart in the cyclic $k$-tuple $B_{l}$.

A $(v, k, \lambda)$ Mendelsohn design $(G, B)$ is said to be $l$-fold perfect if each ordered pair $(x, y)$ of elements of $G$ appears $t$-apart in exactly $\lambda$ of

${ }^{1}$ That is, it admits a regular group of automorphisms which is cyclic. 
the blocks of $B$ for all $t=1,2, \ldots, l$. If $l=k-1$, the design $(G, B)$ is said to be perfect. We call such a design a $(v, k, \lambda)$-PMD.

We show next that Mendelsohn designs which are $l$-fold perfect can be constructed with the aid of generalized complete and near complete mappings which have appropriate properties and we give a number of examples of such constructions.

\section{DEFINITION 5.7. Let}

$$
\left(g_{11} g_{12} \cdots g_{1 k}\right)\left(g_{21} g_{22} \cdots g_{2 k}\right) \cdots\left(g_{s 1} g_{s 2} \cdots g_{s k}\right)
$$

be a $(k, \lambda)$ complete mapping of a group $(G, \cdot)$ of order $v$ such that for each value of $t, t=1,2, \ldots, l$, the elements $g_{i j}^{-1} g_{l, j+t}$ (where $i=1,2, \ldots, s$; and the second suffix $j$ is added modulo $k$ ) comprise the non-identity elements of $G$ each counted $\lambda$ times. Then the complete mapping is said to be an $l$-fold perfect $(k, \lambda)$ complete mapping. If $l=k-1$, the mapping is said to be a perfect $(k, \lambda)$ complete mapping.

We note that a 1 -fold perfect $(k, \lambda)$ complete mapping is a $(k, \lambda)$ complete mapping as given in Definition 2.5 of [10].

DEFINITION 5.8. Let

$$
\begin{aligned}
{\left[g_{11}^{\prime} g_{12}^{\prime} \cdots g_{1 h}^{\prime}\right] } & \cdots\left[g_{\lambda 1}^{\prime} g_{\lambda 2}^{\prime} \cdots g_{\lambda h}^{\prime}\right]\left(g_{11} g_{12} \cdots g_{1 k}\right) \\
& \cdots\left(g_{s 1} g_{s 2} \cdots g_{s k}\right), \quad \text { where } k-h=1,
\end{aligned}
$$

be a $(k, \lambda)$ near complete mapping of a group $(G, \cdot)$ of order $v$ and let $B^{*}$ denote the set of $\lambda+s k$-tuples

$$
\begin{gathered}
\left(g_{11}^{\prime} g_{12}^{\prime} \cdots g_{1 k}^{\prime}\right)\left(g_{21}^{\prime} g_{22}^{\prime} \cdots g_{2 k}^{\prime}\right) \cdots\left(g_{\lambda 1}^{\prime} g_{\lambda 2}^{\prime} \cdots g_{\lambda k}^{\prime}\right)\left(g_{11} g_{12} \cdots g_{1 k}\right) \\
\cdots\left(g_{s 1} g_{s 2} \cdots g_{s k}\right), \quad \text { where } g_{i k}^{\prime}=\infty \text { for } i=1,2, \ldots, \lambda .
\end{gathered}
$$

If, for each value of $t, t=1,2, \ldots, l$, the elements of the set

$$
\left(\bigcup_{i=1}^{\lambda}\left\{g_{i j}^{\prime-1} g_{i, j+t}^{\prime} j=1,2, \ldots, k-1\right\} \cup\left(\bigcup_{i=1}^{s}\left\{g_{l j}^{-1} g_{i, j+t} ; j=1,2, \ldots, k\right\}\right)\right.
$$

comprise all the non-identity elements of the set $G \cup\{\infty\}$ each counted $\lambda$ times where we define $g^{-1} \infty=\infty$, then the near complete mapping is called $l$-fold perfect. If $l=k-1$, the mapping is called perfect.

A 1-fold perfect $(k, \lambda)$ near complete mapping is a $(k, \lambda)$ near complete mapping as given in Definition 2.5 of [10].

THEOREM 5.4. If there exists an l-fold perfect $(k, \lambda)$ complete mapping of a group $(G, \cdot)$ of order $v$ then, by the construction of Theorem 5.1, there exists $a(v, k, \lambda)-M D$ which is $l$-fold perfect and on which the left regular representation $L_{G}$ of $G$ acts as a regular group of automorphisms. Likewise, 
if there exists an l-fold perfect $(k, \lambda)$ near complete mapping of the group then, by the construction of Theorem 5.2, there exists $a(v+1, k, \lambda)-M D$ with these same properties.

Proof. The proof is closely similar to that of Theorems 5.1 and 5.2. We shall prove the second statement of the theorem.

We wish to show that each ordered pair of elements of the set $G \cup\{\infty\}$ occur $t$-apart (for $t=1,2, \ldots, l$ ) $\lambda$ times among the blocks of the set

$$
\begin{aligned}
B=\bigcup_{g \in G}\left\{\left(g g_{11}^{\prime} g g_{12}^{\prime} \cdots\right.\right. & \left.g g_{1, k-1}^{\prime} \infty\right),\left(g g_{21}^{\prime} g g_{22}^{\prime} \cdots g g_{2, k-1}^{\prime} \infty\right), . . \\
\left(g g_{\lambda 1}^{\prime} g g_{\lambda 2}^{\prime} \cdots\right. & \left.g g_{\lambda, k-1}^{\prime} \infty\right),\left(g g_{11} g g_{12} \cdots g g_{1 k}\right), \\
& \left.\left(g g_{21} g g_{22} \cdots g g_{2 k}\right), \ldots,\left(g g_{s 1} g g_{s 2} \cdots g g_{s k}\right)\right\} .
\end{aligned}
$$

Let $(u, w)$ be an ordered pair of elements of $G$. In the given $l$-fold perfect $(k, \lambda)$ near complete mapping of the group $(G, \cdot)$, the element $u^{-1} w$ occurs $\lambda$ times in the form $g_{i j}^{\prime-1} g_{i, j+t}^{\prime}$ or $g_{i j}^{-1} g_{i, j+t}$ for suitable values of $i$ and $j$, where the second suffix is added modulo $k$. Define $g$ as the solution of the equation $g g_{i j}^{*}=u$ where $g_{i j}^{*}$ is the first member of one of the products which is equal to $u^{-1} w$. Then $w=u\left(u^{-1} w\right)=g g_{i, j+t}^{*}$ where $g_{i, j+t}^{*}$ is the second member of the product. It follows that, for this value of $g$, the ordered pair $\left(g g_{i j}^{*}, g g_{i, j+t}^{*}\right)$ is the pair $(u, w)$. So, the pair $(u, w)$, for each choice of $u, w \in G$, occurs $\lambda$ times among the blocks of $B$. We have also to show that each ordered pair $(u, \infty)$ and each ordered pair $(\infty, u)$ for $u \in G$ occur $t$-apart $\lambda$ times. To see this, define $g_{l}$ as the solution of the equation $g_{l} g_{l, k-t}^{\prime}=u(i=1,2, \ldots, \lambda)$. Then $\left(g_{l} g_{i, k-t}^{\prime}, \infty\right)$ is the ordered pair $(u, \infty)$. Since $i$ can take $\lambda$ values, we get $\lambda$ occurrences of this ordered pair in the set of blocks $B$. Finally, define $\bar{g}_{i}$ as the solution of the equation $\bar{g}_{\imath} g_{\imath t}^{\prime}=u(i=1,2, \ldots, \lambda)$. Then $\left(\infty, \bar{g}_{\imath} g_{t t}^{\prime}\right)$ is the ordered pair $(\infty, u)$. Again $i$ can take $\lambda$ values so we get $\lambda$ occurrences of this ordered pair in the set of blocks $B$.

The general problem of constructing $l$-fold perfect generalized complete mappings turns out to be a difficult one. Even for cyclic groups, the problem is not trivial. However, we have obtained a number of examples; in particular, Examples 9, 10, 11 and 12 of the Appendix.

We remark that any $(3, \lambda)$ complete mapping must be perfect. See Theorem 5.6 below.

In [12], an $l$-fold perfect $(v-1,1)$ complete mapping of a group $(G, \cdot)$ of order $v$ has been called an $R_{l}$-sequencing of the group (see also Definition 6.2) and it has been shown in [6], page 237, Theorem 7.4.1, and [11] that if a group $G$ is $R_{l}$-sequenceable then it is possible to construct at 
least $l+1$ mutually orthogonal latin squares based on the Cayley table of $G$. Unfortunately, the problem of deciding which groups are $R_{l}$ sequenceable for a particular value of $l$ seems not to be a simple one. However, we have

THEOREM 5.5. The elementary abelian group of order $p^{n}$ has $a\left(p^{n}-1,1\right)$ complete mapping which is perfect.

Proof. This follows from the fact that such groups are $R_{p^{n}-2^{-}}$ sequenceable. (See [12].)

Computer searches reported in [12] have shown that no 2-fold perfect $(8,1)$ complete mapping of the cyclic group $C_{9}$ exists, that no 3 -fold perfect $(14,1)$ complete mapping of the cyclic group $C_{15}$ exists but that this latter group has 32 isomorphically distinct 2-fold perfect $(14,1)$ complete mappings. (Three of these latter were used to construct our Example 9.)

From Definition 2.5 of [10], it follows that, for an $l$-fold perfect $(k, \lambda)$ complete mapping of a group $(G, \cdot)$ of order $v, k$ divides $\lambda(v-1)$. Consequently, for the corresponding $l$-fold perfect $(v, k, \lambda)$-MD constructed as in Theorem 5.4 we must have $\lambda(v-1) \equiv 0(\bmod k)$. The analogous result for an $l$-fold perfect $(k, \lambda)$ near complete mapping of a group of order $v-1$ is $\lambda v \equiv 0 \bmod k$ since in this case we have $\lambda(k-1)$ $+s k=\lambda(v-1)$, where $s$ is as in Definition 2.4 of [10]. So, for the corresponding $l$-fold perfect $(v, k, \lambda)$-MD constructed as in Theorem 5.4, we have $\lambda v \equiv 0 \bmod k$ in this case.

In [2], a $(v, k, 1)$ resolvable perfect Mendelsohn design is constructed for all sufficiently large $v$ with $v \equiv 1(\bmod k)$. Also, in [13], Mendelsohn has obtained $(v, k, 1)$ perfect Mendelsohn designs with $v(v-1) \equiv 0$ $(\bmod k)$ for the case when $k$ is an odd prime and $v$ is sufficiently large. Theorem 5.4 enlarges the spectrum of $(v, k, \lambda)$ perfect Mendelsohn designs in both these cases. Moreover, the designs obtained admit a regular group of automorphisms and, when $\lambda=1$, they are resolvable.

THEOREM 5.6. Every $(3, \lambda)$ complete or near complete mapping is perfect.

Proof. Let $\left(\begin{array}{lll}g_{11} & g_{12} & g_{13}\end{array}\right)\left(\begin{array}{lll}g_{21} & g_{22} & g_{23}\end{array}\right) \cdots\left(\begin{array}{lll}g_{s 1} & g_{s 2} & g_{s 3}\end{array}\right)$ be a $(3, \lambda)$ complete mapping of a group $(G, \cdot)$ of order $v$. Each pair of elements are either 1-apart or 2-apart. 
Since $g_{i j}^{-1} g_{i, j+2}=\left(g_{i, j+2}^{-1} g_{i j}\right)^{-1}$, where the second suffix is added modulo 3 , and since the elements $g_{1, j+2}^{-1} g_{\imath j}$ comprise the non-identity elements of $G$ each counted $\lambda$ times by definition of a complete mapping, the complete mapping is 2 -fold perfect and so perfect.

Next let $\left[g_{11}^{\prime} g_{12}^{\prime}\right] \cdots\left[g_{\lambda 1}^{\prime} g_{\lambda 2}^{\prime}\right]\left(g_{11} g_{12} g_{13}\right) \cdots\left(g_{s 1} g_{s 2} g_{s 3}\right)$ be a $(3, \lambda)$ near complete mapping. The elements $g_{i 1}^{\prime-1} g_{i 2}^{\prime}$ and $g_{i j}^{-1} g_{i, j+1}($ for $j=1,2)$ together with the elements $g_{i 3}^{-1} g_{i 1}$ comprise the non-identity elements of $G$ each counted $\lambda$ times. Consequently, so also do the elements $g_{i 2}^{\prime-1} g_{i 1}^{\prime}$ and $g_{i, j+1}^{-1} g_{i j}$ for $(j=1,2)$ together with the elements $g_{i 1}^{-1} g_{i 3}$. From this, we see at once that the set $B^{*}$ of $\lambda+s$ triples $\left(g_{11}^{\prime} g_{12}^{\prime} \infty\right) \cdots\left(g_{\lambda 1}^{\prime} g_{\lambda 2}^{\prime} \infty\right)\left(g_{11} g_{12}\right.$ $\left.g_{13}\right) \cdots\left(g_{s 1} g_{s 2} g_{s 3}\right)$ satisfies the condition given in Definition 5.8 for the near complete mapping to be 2 -fold perfect and so perfect.

From Theorem 5.6, it follows that sufficient conditions for the existence of a $(v, 3, \lambda)$-PMD are the existence of a $(3, \lambda)$ complete mapping of a group of order $v$ or of a $(3, \lambda)$ near complete mapping of a group of order $v-1$. In either case the perfect Mendelsohn design admits the group $G$ as a regular group of automorphisms. (See Example 13.)

6. Constructions for generalized complete mappings. In this final section, we provide a number of different constructions for generalized complete and near complete mappings.

We begin with the following simple observations:

(i) If we repeat the cycles and sequences of a $(K, \lambda)$ generalized complete or near complete mapping $h$ times, we get an $(h K, h \lambda)$ generalized complete mapping. Such a construction is called trivial and the generalized complete mapping so obtained is called trivially decomposable.

(ii) If we reverse the order of the elements in all the cycles and sequences of a generalized complete or near complete mapping, we get another such mapping. Moreover, if

$$
\begin{array}{r}
{\left[\begin{array}{llll}
g_{11}^{\prime} g_{12}^{\prime} & \cdots & g_{1 h_{1}}^{\prime}
\end{array}\right] \cdots\left[\begin{array}{lll}
g_{\lambda 1}^{\prime} g_{\lambda 2}^{\prime} \cdots & g_{\lambda h_{\lambda}}^{\prime}
\end{array}\right]\left(g_{11} g_{12} \cdots g_{1 k_{1}}\right)} \\
\cdots\left(g_{s 1} g_{s 2} \cdots g_{s k_{s}}\right)
\end{array}
$$

is a $(K, \lambda)$ near complete mapping which satisfies the condition

$$
\begin{aligned}
& \left\{g_{l j}^{\prime-1} g_{i, j+1}^{\prime}: i=1,2, \ldots, \lambda ; j=1,2, \ldots, h_{i}\right\} \\
& \quad=\left\{g_{l, j+1}^{\prime-1} g_{l j}^{\prime}: i=1,2, \ldots, \lambda ; j=1,2, \ldots, h_{l}\right\},
\end{aligned}
$$

then the mapping

$$
\begin{array}{r}
{\left[\begin{array}{llll}
g_{11}^{\prime} g_{12}^{\prime} \cdots & g_{1 h_{1}}^{\prime}
\end{array}\right] \cdots\left[g_{\lambda 1}^{\prime} g_{\lambda 2}^{\prime} \cdots g_{\lambda h_{\lambda}}^{\prime}\right]\left(g_{1 k_{1}} g_{1, k_{1}-1} \cdots g_{11}\right)} \\
\cdots\left(g_{s k_{s}} g_{s, k_{s}-1} \cdots g_{s 1}\right)
\end{array}
$$

is another near complete mapping. (See Example 14.) 
(iii) If we adjoin a $(K, \lambda)$ generalized complete mapping to its reverse, we get a $(2 K, 2 \lambda)$ generalized mapping. A mapping which can be so constructed is called patterned.

A generalized complete mapping obtained from a given generalized complete mapping by the methods of (ii) above has the parameters $K$ and $\lambda$ unchanged. However, this invariant property is combinatorial rather than algebraic because this type of transformation does change the properties of the corresponding neofield. (See Examples 15 and 16.)

A construction very similar to that of (ii) which yields a $(v+1,2)$ generalized near complete mapping of a group $G$ of order $v$ if $G$ is quasisequenceable is the following. Suppose that $b_{0}, b_{1}, b_{2}, \ldots, b_{v-1}$ is the terrace associated with the quasisequencing (see [1] for the definitions). It has the property that the $v-1$ elements $b_{0}^{-1} b_{1}, b_{1}^{-1} b_{2}, \ldots, b_{v-2}^{-1} b_{v-1}$ contain each element of order 2 of $G$ once and, for each other non-identity element of $G$, either $g$ twice or $g^{-1}$ twice or both $g$ and $g^{-1}$. Consequently, $\left[b_{0} b_{1} b_{2} \cdots b_{v-1}\right]\left[b_{v-1} b_{v-2} \cdots b_{0}\right]$ is a $(v+1,2)$ generalized near complete mapping of $G$. It can be expressed in canonical form by multiplying the elements of the first sequence by $b_{0}^{-1}$ and those of the second by $b_{v-1}^{-1}$. Moreover, if $b_{v-1}^{-1} b_{0}=b_{i}^{-1} b_{i+1}$ then

$$
\left[b_{0} b_{1} b_{2} \cdots b_{v-1}\right]\left[b_{\imath} b_{i-1} \cdots b_{0} b_{v-1} b_{v-2} \cdots b_{i+2} b_{\imath+1}\right]
$$

also is a $(v+1,2)$ generalized complete mapping. (Compare page 329 of [1].)

A slightly less obvious way of constructing new generalized complete mappings comes from the observation that, if a group $G$ can be expressed as a direct product $G=G_{1} \times G_{2}$ then a complete mapping of $G$ can be constructed from complete mappings of its direct factors $G_{1}$ and $G_{2}$. Precisely, we have

THEOREM 6.2. If there exists a $\left(k_{1}, \lambda_{1}\right)$ complete mapping of the group $G_{1}$ and $a\left(k_{2}, \lambda_{2}\right)$ complete mapping of the group $G_{2}$, then there exists $a$ $(K, \lambda)$ complete mapping of the group $G_{1} \times G_{2}$, where $\lambda=\lambda_{1} \cdot \lambda_{2}, K=$ $\left\{k, k, \ldots, k, k_{1}, k_{1}, \ldots, k_{1}, k_{2}, k_{2}, \ldots, k_{2}\right\}$ and $k$ is the least common multiple of $k_{1}$ and $k_{2}$.

Proof. Suppose that

$$
\boldsymbol{\sigma}_{i}=\left(g_{11}^{(i)} g_{12}^{(i)} \cdots g_{1 k_{t}}^{(i)}\right)\left(g_{21}^{(i)} g_{22}^{(i)} \cdots g_{2 k_{t}}^{(i)}\right) \cdots\left(g_{s_{t}, 1}^{(i)} g_{s_{t}, 2}^{(i)} \cdots g_{s_{t}, k_{t}}^{(i)}\right)
$$

is a $\left(k_{i}, \lambda_{i}\right)$ complete mapping of $G_{i}, i=1,2$. Let $G_{i}^{*}$ denote the set of non-identity elements of $G_{i}$. Then we may regard $\sigma_{i}$ as a permutation from $\lambda_{\imath} G_{i}^{*}$ to $\lambda_{i} G_{i}^{*}$ with cyclic structure $\left\{k_{l}, k_{i}, \ldots, k_{\imath}\right\}$ and such that $\sigma_{i}\left(g_{j, l}^{(l)}\right)$ $=g_{J, l+1}^{(i)}$. Here, the second suffix is added modulo $k_{l}$ and $\lambda_{i} G_{l}^{*}$ is a set 
which consists of the non-identity elements of $G_{i}$ each counted $\lambda$ times. The permutation $\sigma_{i}$ satisfies the extra condition that the set $\left\{g^{-1} \sigma_{i}(g)\right.$ : $\left.g \in \lambda_{i} G_{l}^{*}\right\}$ coincides with the set $\lambda_{t} G_{l}^{*}$.

We shall obtain the desired $(K, \lambda)$ complete mapping of $G_{1} \times G_{2}$ by constructing its associated permutation $\sigma$.

We define $\sigma_{t}\left(e_{i}\right)=e_{l}$, where $e_{i}$ is the identity element of $G_{i}$. Let $\sigma$ be the permutation from $\lambda\left(G_{1} \times G_{2}\right) *$ to $\lambda\left(G_{1} \times G_{2}\right)^{*}$ defined by $\sigma\left(g_{1}, g_{2}\right)$ $=\left(\sigma_{1}\left(g_{1}\right), \sigma_{2}\left(g_{2}\right)\right)$ where $\left(g_{1}, g_{2}\right) \in \lambda\left(G_{1} \times G_{2}\right)^{*}$. Thus,

$$
\sigma\left(g_{j_{1} l_{1}}^{(1)}, g_{j_{2} l_{2}}^{(2)}\right)=\left(g_{j_{1}, l_{1}+1}^{(1)}, g_{j_{2}, l_{2}+1}^{(2)}\right), \quad \sigma\left(g_{j_{1} l_{1}}^{(1)}, e_{2}\right)=\left(g_{J_{1}, l_{1}+1}^{(2)}, e_{2}\right)
$$

and

$$
\sigma\left(e_{1}, g_{J_{2} l_{2}}^{(2)}\right)=\left(e_{1}, g_{j_{2}, l_{2}+1}^{(2)}\right) \text {. }
$$

From this it is immediately clear that if $\left(g_{1}, g_{2}\right)$ is an element of $\lambda\left(G_{1} \times G_{2}\right)^{*}$ for which $g_{1} \neq e_{1}$ and $g_{2} \neq e_{2}$ then each of the $\lambda=\lambda_{1} \cdot \lambda_{2}$ cycles in which $\left(g_{1}, g_{2}\right)$ appears is of length $k=\left[k_{1}, k_{2}\right]$, where $\left[k_{1}, k_{2}\right]$ denotes lowest common multiple. If $g_{1}=e_{1}, g_{2} \neq e_{2}$, then each of the $\lambda$ cycles in which $\left(g_{1}, g_{2}\right)$ appears is of length $k_{2}$ and these cycles comprise $\lambda_{1}$ copies of a set of cycles isomorphic to those of the generalized complete mapping $\sigma_{2}$. A similar remark applies to the case $g_{1} \neq e_{1}, g_{2}=e_{2}$. Finally, it is easy to check that there are all together $\left(k_{1} k_{2} / k\right) s_{1} s_{2}$ cycles of length $k=\left[k_{1}, k_{2}\right], \lambda_{2} s_{1}$ cycles of length $k_{1}$ and $\lambda_{1} s_{2}$ cycles of length $k_{2}$, whence the set $\left\{g^{-1} \sigma(g): g \in \lambda\left(G_{1} \times G_{2}\right)^{*}\right\}$ has cardinal $s_{1} s_{2} k_{1} k_{2}+\lambda_{2} s_{1} k_{1}+$ $\lambda_{1} s_{2} k_{2}$. It is also easy to check that this set coincides with the set $\lambda\left(G_{1} \times G_{2}\right)^{*}$ and so $\sigma$ is a generalized complete mapping of $G_{1} \times G_{2}$. [Note that ord $G_{i}=1+s_{i} k_{l} / \lambda_{l}$, whence

$$
\text { ord } G^{*}=\left(1+s_{1} k_{1} / \lambda_{1}\right)\left(1+s_{2} k_{2} / \lambda_{2}\right)-1
$$

and so $\lambda G^{*}$ has cardinality $\left(\lambda_{1}+s_{1} k_{1}\right)\left(\lambda_{2}+s_{2} k_{2}\right)-\lambda_{1} \lambda_{2}$.]

Since the direct product of two cyclic groups is cyclic if and only if the orders of the two cyclic groups are relatively prime, we can use Theorem 6.1 to construct generalized mappings of a cyclic group $C_{n}$ from those of cyclic groups of smaller orders provided that these orders are relatively prime factors of $n$.

In the Appendix, we give two illustrations of the theorem which emphasize this (Examples 17 and 18).

The investigation of methods for constructing $(K, \lambda)$ generalized complete mappings with $\lambda>1$ was first initiated in [9], page 43, where certain $(K, 2)$ generalized complete mappings of a cyclic group $C_{n}$ were constructed. It was shown (in Chapter 3 of [9]) that there exist $(6,2)$ complete mappings of $C_{n}$ for $n \equiv 1,7$ or $13(\bmod 18)$; that there exist 
$(K, 2)$ complete mappings of $C_{n}$ for $n \equiv 3(\bmod 18)$ with $K=$ $\{2,2,6,6, \ldots, 6\}$ and that there exist $(K, 2)$ near complete mappings of $C_{n}$ for $n \equiv 2,8$ or $14(\bmod 18)$ with $K=\{2,2 ; 6,6, \ldots, 6\}$. It was also shown that the cyclic neofields associated with these generalized complete mappings (see Theorem I.46 in [9]) all have the XIP-property. (A cyclic neofield $N$ is said to have the crossed-inverse property (XIP-property) if $(x+y)+(-x)=y$, for all $x, y \in N)$. For illustration, see Examples 19 to 23 .

Example 19 is different from Examples 20 and 22 in the sense that if we consider the cyclic sequences in each example as subsets of the respective groups then each of Examples 20 and 22 has two equal subsets. This suggests the following definition.

Definition 6.1. A $(K, \lambda)$ generalized complete mapping, $\lambda>1$, of the group $G$ is said to be tight if, when we ignore the cyclic order in each cyclic sequence (i.e. treat the sequences as subsets of the group $G$ ), all of the cyclic sequences are distinct.

The generalized complete mappings of Examples 20 and 22 are clearly not tight. The general problem of obtaining tight (and consequently non-patterned and not trivially decomposable) generalized complete mappings for a given group is difficult. So far, the only known family of tight generalized complete mappings are $(6,2)$ complete mappings of the cyclic group $C_{n}$ for $n \equiv 1,13(\bmod 18)$ (see [9], page 80$)$.

One method of obtaining $(\lambda K, \lambda)$ tight generalized complete mappings is to construct $\lambda$ distinct (in the sense implied by Definition 6.1) $(K, 1)$ generalized complete mappings and then adjoin them. Here $\lambda K$ is obtained from $K$ by repeating each element of $K \lambda$ times. As an example, we have that

$$
\left[e a^{4} a^{3}\right]\left[\begin{array}{lll}
e & a^{2} & a^{3}
\end{array}\right]\left[\text { e } a a^{3}\right]\left[\begin{array}{lll}
5 & a^{5} & a^{3}
\end{array}\right]\left(a a^{2} a^{5}\right)\left(a a^{5} a^{4}\right)\left(a^{2} a^{5} a^{4}\right)\left(a a^{2} a^{4}\right)
$$

is a tight $(4 K, 4)$ near complete mapping of the cyclic group $C_{6}$ obtained by putting four $(K, 1)$ near complete mappings together, where $K=\{3 ; 3\}$ and $4 K=\{3,3,3,3 ; 3,3,3,3\}$.

The following theorem gives a construction which always yields tight generalized complete mappings. We first need a definition. In part I we defined an $R$-sequencing of a group. The following generalization was first introduced in [12].

Definition 6.2. A group $(G, \cdot)$ of order $n$ is said to be $R_{l}$-sequenceable if $n-1$ of its elements (which we may take to be the non-identity elements) can be arranged in a sequence $c_{1}, c_{2}, \ldots, c_{n-1}$ in such a way that 
the set of elements $c_{i}^{-1} c_{i+1}$ for $i=1,2, \ldots, n-1$, are all distinct (where arithmetic of suffices is modulo $n-1)$ and likewise the sets of elements $c_{i}^{-1} c_{i+2}, c_{l}^{-1} c_{i+3}, \ldots, c_{i}^{-1} c_{i+l}$. In particular, a group which is $R_{l}$-sequenceable is $R$-sequenceable with $a_{0}=e$, and $a_{1}=c_{n-1}^{-1} c_{1}, a_{2}=c_{1}^{-1} c_{2}, a_{3}=$ $c_{2}^{-1} c_{3}, \ldots, a_{n-1}=c_{n-2}^{-1} c_{n-1}$. (Compare Definition 2.2 of [10].)

An $R_{l}$-sequencing of a group is the same as an $l$-fold perfect $(v-1,1)$ complete mapping of that group as we mentioned in the previous section.

TheOREM 6.2. If a group $(G, \cdot)$ of order $n$ is $R_{\text {r-sequenceable then it }}$ possesses a tight $(K, \lambda)$ complete mapping where $\lambda$ is equal to the number of integers in the set $\{(n-1) / i: i=1,2, \ldots, l\}$.

Proof. In the notation of Definition 6.2, the mapping $\sigma=\left(c_{1} c_{2} \cdots\right.$ $\left.c_{n-1}\right)$ is an $(n-1,1)$ complete mapping of $G$. (Compare Theorem 2.1 (iii) of [10].) Since $G$ is $R_{r}$-sequenceable, $\sigma^{2}, \sigma^{3}, \ldots, \sigma^{l}$ are also complete mappings of $G$. The cyclic sequences of $\sigma^{i}$ and $\sigma^{j}$ are different from each other and from the cyclic sequences of $\sigma$ provided that $i$ and $j$ are distinct divisors of $n-1$. By adjoining these complete mappings, we get a tight generalized complete mapping of the kind described in the theorem.

It was shown in [12] that an elementary abelian group of order $p^{n}$ is $R_{\digamma}$-sequenceable for $l=1,2, \ldots, p^{n}-2$. Making use of this fact, we obtain Examples 24 and 25.

We remark that, for any elementary abelian group of order $p^{n}$, Theorem 6.2 also provides a simple method of constructing $(k, 1)$ complete mappings where $k$ is any integer which divides $n-1$.

Definition 6.3. A $(K, \lambda)$ complete mapping $\left(g_{11} g_{12} \cdots g_{1 k_{1}}\right)\left(g_{21}\right.$ $\left.g_{22} \cdots g_{2 k_{2}}\right) \cdots\left(g_{s 1} g_{s 2} \cdots g_{s k_{s}}\right)$ of a group $(G, \cdot)$ is said to be strong if the elements $g_{\imath j} g_{\imath, j+1}$ (where $i=1,2, \ldots, s$; and the second suffix $j$ is added modulo $k_{\imath}$ ) comprise the non-identity elements of $G$ each counted $\lambda$ times. Similarly, a $(K, \lambda)$ near complete mapping

$$
\begin{array}{r}
{\left[\begin{array}{llll}
g_{11}^{\prime} g_{12}^{\prime} \cdots & g_{1 h_{1}}^{\prime}
\end{array}\right] \cdots\left[\begin{array}{lll}
g_{\lambda 1}^{\prime} & g_{\lambda 2}^{\prime} \cdots & \left.g_{\lambda h_{\lambda}}^{\prime}\right]
\end{array}\right]} \\
\cdots\left(g_{11} g_{12} \cdots g_{1 k_{1}}\right) \\
\cdots\left(g_{s 1} g_{s 2} \cdots g_{s k_{s}}\right)
\end{array}
$$

of the group is said to be strong if the elements $g_{i j}^{\prime} g_{i, j+1}^{\prime}$ and $g_{i j} g_{i, j+1}$ together with the elements $g_{i k_{l}} g_{i 1}$ comprise the non-identity elements of $G$ each counted $\lambda$ times.

The concept of "strongness" of a complete mapping was first introduced in [8], where (among other things) a special case of the following theorem applicable only to cyclic groups and to the particular value $k=3$ was proved. 
THEOREM 6.3. Let $(G, \cdot)$ be a finite group. If there exists a strong $(2 k, \lambda)$ complete mapping of $G$ which satisfies the condition that, for each cyclic sequence $\left(g_{\imath 1} g_{i 2} \cdots g_{\imath, 2 k}\right), g_{l j}^{-1} g_{\imath, j+1}=g_{l, j+2}$ where the second suffix is added modulo $2 k$, then there exists $a(k, \lambda)$ complete mapping of $G$.

Proof. Let $\sigma=\left(g_{11} g_{12} \cdots g_{1,2 k}\right) \cdots\left(g_{s 1} g_{s 2} \cdots g_{s, 2 k}\right)$ be a strong $(2 k, \lambda)$ complete mapping of the group $G$ such that $g_{i j}^{-1} g_{i, J+1}=g_{i, J+2}$. Then the mapping

$$
\boldsymbol{\sigma}^{2}=\left(g_{11} g_{13} \cdots g_{1,2 k-1}\right)\left(g_{12} g_{14} \cdots g_{1,2 k}\right) \cdots\left(g_{s 2} g_{s 4} \cdots g_{s, 2 k}\right)
$$

is the desired $(k, \lambda)$ complete mapping because we have that

$$
g_{l j}^{-1} g_{i, j+2}=\left(g_{l j}^{-1} g_{i, j+1}\right)\left(g_{l, j+1}^{-1} g_{i, j+2}\right)=g_{\imath, j+2} g_{i, j+3}
$$

and, because $\sigma$ is strong, the products $g_{l, j+2} g_{i, j+3}$ comprise the non-identity elements of $G$ each counted $\lambda$ times. (Arithmetic of all second suffices is modulo $2 k$.)

We remark that if $(G,+)$ is an elementary abelian group of order $p^{n}$, we may regard it as the additive group of the Galois field GF $\left[p^{n}\right]$. If $\omega$ is a primitive element of $\mathrm{GF}\left[p^{n}\right]$ then $\sigma=\left(1 \omega \omega^{2} \cdots \omega^{p^{n}-2}\right)$ is an $R_{\digamma}$-sequencing of $(G,+)$, where $l=p^{n}-2$. So, if $2 k$ is any even divisor of $p^{n}-1$, and $t=\left(p^{n}-1\right) / 2 k$ we have that

$$
\begin{aligned}
& \sigma^{t}=\left(1 \omega^{t} \omega^{2 t} \cdots \omega^{(2 k-1) t}\right)\left(\omega \omega^{t+1} \omega^{2 t+1}\right.\left.\cdots \omega^{(2 k-1) t+1}\right) \\
& \cdots\left(\omega^{t-1} \omega^{2 t-1} \cdots \omega^{2 k t-1}\right)
\end{aligned}
$$

is a $(2 k, 1)$ complete mapping of $(G,+)$. Also $\sigma^{t}$ is strong because the elements $1+\omega^{t}, \omega+\omega^{t+1}, \ldots, \omega^{t-1}+\omega^{2 t-1}, \omega^{t}+\omega^{2 t}, \omega^{t+1}+$ $\omega^{2 t+1}, \ldots, \omega^{2 t-1}+\omega^{3 t-1}, \ldots, \omega^{(2 k-1) t-1}+\omega^{2 k t-1}$ are the non-identity elements of $G$. It satisfies the further condition required by Theorem 6.3 if and only if $\omega^{t}-1 \equiv \omega^{2 t}(\bmod p)$. However, in this special case, $\sigma^{2 t}$ is a $(k, 1)$ complete mapping of $(G,+)$ even when this extra condition is not satisfied.

Example 26 is constructed by the method just described and satisfies the additional condition $\omega^{t}-1 \equiv \omega^{2 t}(\bmod 13)$ where $\omega=2$ and $t=2$. Example 27 is constructed from the Galois field GF[9] by the method just described but does not satisfy the additional condition $\omega^{t}-1 \equiv \omega^{2 t}$. That is, $\alpha^{2}-1 \not \equiv \alpha^{4}(\bmod 3)$.

A final observation concerning Theorem 6.3 is that, if the group $G$ is abelian, the condition $g_{l j}^{-1} g_{i, j+1}=g_{i, j+2}$ can only hold when $k=6$. Let $a, b$ be two elements of such a group. Then the cyclic sequence $(a b \cdots)$ must continue as follows: $a, b, a^{-1} b, b^{-1}\left(a^{-1} b\right)=a^{-1},\left(a^{-1} b\right)^{-1} a^{-1}=b^{-1}$, $a b^{-1}, a, b$. That is, it has length six. 
The following is an analogue of Theorem 6.3 for near complete mappings.

THEOREM 6.4. Let $(G, \cdot)$ be a finite group and let

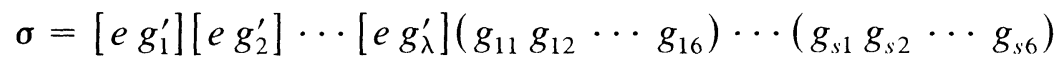

be $a(K, \lambda)$ near complete mapping of $(G, \cdot)$, where $K=\{2,2, \ldots, 2$; $6,6, \ldots, 6\}$. If $\sigma$ is strong and satisfies the condition that, for each cyclic sequence $\left(g_{i 1} g_{i 2} \cdots g_{i 6}\right), g_{i j}^{-1} g_{t, 1+1}=g_{t, j+2}$ where the second suffix is added modulo 6 , then there exists a $(3, \lambda)$ near complete mapping of $G$.

Proof. The mapping

$$
\begin{aligned}
\boldsymbol{\sigma}^{*}= & {\left[\text { e } g_{1}^{\prime}\right]\left[\text { e } g_{2}^{\prime}\right] \cdots\left[\text { e } g_{\lambda}^{\prime}\right]\left(g_{11} g_{13} g_{15}\right)\left(g_{12} g_{14} g_{16}\right) } \\
& \cdots\left(g_{s 1} g_{s 3} g_{s 5}\right)\left(g_{s 2} g_{s 4} g_{s 6}\right)
\end{aligned}
$$

is the desired $(3, \lambda)$ near complete mapping because for the cyclic sequences we have

$$
g_{i,}^{-1} g_{\imath, j+2}=\left(g_{\imath j}^{-1} g_{\imath, j+1}\right)\left(g_{i, j+1}^{-1} g_{\imath, j+2}\right)=g_{i, j+2} \cdot g_{\imath, j+3}
$$

and because $\sigma$ is strong the products $g_{t, j+2} g_{t, j+3}$ together with the products $e g_{1}^{\prime}, e g_{2}^{\prime}, \ldots, e g_{\lambda}^{\prime}$ comprise the non-identity elements of $G$ each counted $\lambda$ times.

\section{APPENDIX-EXAMPLES.}

(1) Let $G=C_{14} \cup\{\infty\}$ and $B=\bigcup_{i=0}^{13}\left\{\left(\begin{array}{lll}i & 7+i & \infty\end{array}\right),(1+i 2+i\right.$ $6+i 11+i \quad 3+i),(4+i \quad 12+i \quad 9+i \quad 5+i),(8+i \quad 10+i$ $13+i)\}$, where all addition is modulo 14 . Then $(G, B)$ is a $(15, K, 1)$-MD with $K=\{5,4,3\}$.

(2) Let $G=C_{13}$ and $B=\bigcup_{i=0}^{12}\left\{\left(\begin{array}{lllll}1+i & 4+i & 3+i & 12+i & 9+i\end{array}\right.\right.$ $10+i),(2+i \quad 6+i \quad 4+i \quad 11+i \quad 7+i \quad 9+i), \quad(5+i \quad 2+i$ $11+i \quad 7+i \quad 9+i), \quad(5+i \quad 2+i \quad 10+i \quad 8+i \quad 11+i \quad 3+i)$, $(6+i 1+i 8+i 7+i 12+i \quad 5+i)\}$, where addition is modulo 13 . Then $(G, B)$ is a $(13, K, 2)-\mathrm{MD}$ with $K=\{6\}$. That is, it is a $(13,6,2)$-MD.

(3) Let $G=\{a, b, c, d\}$ and $B=\{(b c d),(a d c),(d a b),(c b a)\}$. Then $(G, B)$ is a $(4, K, 1)-\mathrm{MD}$ with $K=\{3\}$. That is, it is a $(4,3,1)-\mathrm{MD}$.

(4) Let $G$ be the dihedral group $D_{4}$ of order 8 with generating relations $a^{4}=b^{2}=e$ and $a b=b a^{-1}$, and let $B=\bigcup_{x \in G}\left\{\left(x a x a^{2} x b a^{2} x b a\right.\right.$ $\left.\left.x b a^{3} x a^{3} x b\right)\right\}$. Then $(G, B)$ is an $(8,7,1)$-MD. 
(5) Let $G=C_{8} \cup\{\infty\}$ and $B=\cup_{i=0}^{7}\left\{\left(\begin{array}{lll}i & 4+i & \infty\end{array}\right),\left(\begin{array}{ll}i & 4+i\end{array}\right.\right.$ $\infty), \quad(1+i \quad 2+i 7+i), \quad(3+i \quad 6+i \quad 5+i), \quad(1+i \quad 7+i$ $6+i),(2+i \quad 3+i \quad 5+i)\}$, where addition is modulo 8 . Then $(G, B)$ is a $(9,3,2)-\mathrm{MD}$.

(6) The $R$-sequencing e $a b a^{3} a^{2} b a^{2} b a^{3} b a$ of the dihedral group $D_{4}$ defines the $(8,7,1)$-MD whose blocks are given by left-multiplying the elements of the basis blocks by each of the eight elements of $D_{4}$ in turn. Here there is exactly one basis block ( $\left.a a^{2} b a^{2} b a b a^{3} a^{3} b\right)$ by Theorem 2.1(iii) of [10] and hence we get a design with eight blocks.

(7) The $(K, 2)$ near complete mapping of the dihedral group $D_{3}$ given in Example 2.6 of $[\mathbf{1 0}]$, where $K=\{2,2 ; 4,4\}$, defines a $\left(7, K^{*}, 2\right)$-MD with $K^{*}=\{3,4\}$ whose blocks are

$\begin{array}{llll}(e b a \infty), & \left(e b a^{2} \infty\right), & \left(a^{2} b b a^{2} a\right), & \left(a b b a a^{2}\right) \\ (a b \infty), & (a b a \infty), & \left(e b a^{2} b a a^{2}\right), & \left(a^{2} b a^{2} b e\right) \\ \left(a^{2} b a^{2} \infty\right), & \left(a^{2} b \infty\right), & (a b a b e), & \left(e b a b a^{2} a\right) \\ (b a \infty), & \left(b a^{2} \infty\right), & \left(b a^{2} e a^{2} b a\right), & \left(b a e a b a^{2}\right) \\ (b a e \infty), & (b a a \infty), & \left(b a^{2} a b a^{2}\right), & \left(b a^{2} a^{2} e b\right) \\ \left(b a^{2} a \infty\right), & \left(b a^{2} e \infty\right), & (b a a e b), & \left(b a a^{2} b a\right)\end{array}$

The design admits a regular group of automorphisms isomorphic to $D_{3}$.

(8) The sequencing 0321 of the cyclic group $C_{4}$ defines the $(5,5,1)$-MD whose blocks are ( $\left(\begin{array}{lllll}0 & 3 & 1 & 2 & \infty\end{array}\right),\left(\begin{array}{lllll}1 & 0 & 2 & 3 & \infty\end{array}\right),\left(\begin{array}{llll}2 & 1 & 3 & 0\end{array}\right)$ and

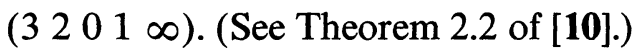

(9) The $(14,3)$ complete mapping of the cyclic group $C_{15}$ given below is 2-fold perfect.

$(1213411103685914127)(1381413211451297106)$ (1492314810751161312).

(10) The $(6,2)$ complete mapping (1 $\left.3 \begin{array}{lllll}1 & 2 & 6 & 4 & 5\end{array}\right)\left(\begin{array}{llllll}1 & 5 & 4 & 6 & 2 & 3\end{array}\right)$ of the cyclic group $C_{7}$ is perfect.

(11) The $(7,1)$ near complete mapping [ $\left[\begin{array}{llllll}0 & 2 & 1 & 4 & 5 & 3\end{array}\right]$ of the cyclic group $C_{6}$ is perfect.

(12) The $(3,2)$ near complete mapping of the cyclic group $C_{14}$ given below is perfect

[0 7] [0 7] (1 2 5) (3 9 4) (6 10 12) (8 13 11) (1 11 9) (2 13 12) (3 68 ) (4 5 10). 
(13) The existence of the following $(3,2)$ near complete mapping of the quaternion group $Q=\operatorname{gp}\left\{a, b: a^{4}=e, a^{2}=b^{2}, a b=b a^{-1}\right\}$ allows the construction of a $(9,3,2)$-PMD admitting this group as a regular group of automorphisms.

$$
[e a]\left[e a^{3}\right]\left(b a b a^{3} a\right)\left(b b a^{2} a^{3}\right)\left(b a a^{2} b a^{2}\right)\left(b a^{2} b a^{3}\right) \text {. }
$$

(14) $\left[e b a^{4}\right]\left(a^{2} a a^{4}\right)\left(a^{3} b a^{2} b a^{6}\right)\left(a^{6} b b a\right)\left(a^{5} b a^{3} b a^{5}\right)$ is a $(3,1)$ near complete mapping of the dihedral group $D_{7}=\operatorname{gp}\left\{a, b: a^{7}=b^{2}=e\right.$, $\left.a b=b a^{-1}\right\}$. By reversing the cyclic order of the elements in each cyclic sequence, the following is also a $(3,1)$ near complete mapping of $D_{7}$ :

$$
\left[e b a^{4}\right]\left(a^{2} a^{4} a\right)\left(a^{3} b a^{6} b a^{2}\right)\left(a^{6} b a b\right)\left(a^{5} b a^{5} b a^{3}\right) \text {. }
$$

(15) $\left(a a^{4} a^{2}\right)\left(a^{3} a^{5} a^{6}\right)$ is a $(3,1)$ complete mapping of the cyclic group $C_{7}$. By observation (ii) of $\S 6,\left(a a^{2} a^{4}\right)\left(a^{3} a^{6} a^{5}\right)$ is also a $(3,1)$ complete mapping of $C_{7}$. By Theorem 3.1 of [10], their associated neofields of order 8 are:

\begin{tabular}{c|cccccccc}
$z$ & 0 & 1 & $a$ & $a^{2}$ & $a^{3}$ & $a^{4}$ & $a^{5}$ & $a^{6}$ \\
\hline$\phi(z)$ & 1 & 0 & $a^{4}$ & $a$ & $a^{5}$ & $a^{2}$ & $a^{6}$ & $a^{3}$
\end{tabular} and

One can easily check that the former neofield hs only the commutativity property: $x+y=y+x$, while the latter has only the right inverse property: $(x+y)(-y)=x($ see [9]).

(16) The following two cyclic neofields of order 9 (associated with two near complete mappings of the cyclic group $C_{8}$ related as in observation (ii) of §6):

\begin{tabular}{c|ccccccccc}
$z$ & 0 & 1 & $a$ & $a^{2}$ & $a^{3}$ & $a^{4}$ & $a^{5}$ & $a^{6}$ & $a^{7}$ \\
\hline$\phi(z)$ & 1 & $a^{4}$ & $a^{7}$ & $a^{3}$ & $a^{5}$ & 0 & $a^{2}$ & $a$ & $a^{6}$
\end{tabular} and

both have the left inverse property: $(-x)+(x+y)=y$. However the first neofield also possesses the commutative property. In fact, it is the Galois field of order 9.

(17) From the $(2,1)$ complete mapping $\sigma_{1}=\left(a a^{2}\right)$ of the cyclic group $C_{3}=\operatorname{gp}\{a\}$ and the $(3,1)$ complete mapping $\sigma_{2}=\left(b b^{2} b^{4}\right)\left(b^{3} b^{6} b^{5}\right)$ of 
the cyclic group $C_{7}=\operatorname{gp}\{b\}$, we can construct the following $(K, 1)$ complete mapping of the direct product $C_{3} \times C_{7}$, where $K=\{6,6,3,3,2\}$. We can conveniently express this in the form of a complete mapping of $C_{21}=\operatorname{gp}\{c\}$ by means of the mapping $\left(a^{x}, b^{y}\right) \rightarrow c^{7 x+3 y}$.

$$
\begin{aligned}
& \left((a, b),\left(a^{2}, b^{2}\right),\left(a, b^{4}\right),\left(a^{2}, b\right),\left(a, b^{2}\right),\left(a^{2}, b^{4}\right)\right) \\
& \left(\left(a, b^{3}\right),\left(a^{2}, b^{6}\right),\left(a, b^{5}\right),\left(a^{2}, b^{3}\right),\left(a, b^{6}\right),\left(a^{2}, b^{5}\right)\right) \\
& \left((e, b),\left(e, b^{2}\right),\left(e, b^{4}\right)\right)\left(\left(e, b^{3}\right),\left(e, b^{6}\right),\left(e, b^{5}\right)\right) \\
& \left((a, e),\left(a^{2}, e\right)\right)
\end{aligned}
$$

(18) From the $(2,1)$ complete mapping $\sigma_{1}=\left(a a^{4}\right)\left(a^{2} a^{3}\right)$ and the $(4,2)$ patterned complete mapping $\sigma_{2}=\left(b b^{3} b^{4} b^{2}\right)\left(b^{2} b^{4} b^{3} b\right)$ of the cyclic group $C_{5}$, we can construct the following $(K, 2)$ complete mapping of the non-cyclic group $C_{5} \times C_{5}$, where $K=\{4,4, \ldots, 4,2,2,2,2\}$.

$$
\begin{aligned}
& \left((a, b),\left(a^{4}, b^{3}\right),\left(a, b^{4}\right),\left(a^{4}, b^{2}\right)\right)\left((a, b),\left(a^{4}, b^{2}\right),\left(a, b^{4}\right),\left(a^{4}, b^{3}\right)\right) \\
& \left(\left(a^{2}, b\right),\left(a^{3}, b^{3}\right),\left(a^{2}, b^{4}\right),\left(a^{3}, b^{2}\right)\right)\left(\left(a^{2}, b\right),\left(a^{3}, b^{2}\right),\left(a^{2}, b^{4}\right),\left(a^{3}, b^{3}\right)\right) \\
& \left(\left(a^{3}, b\right),\left(a^{2}, b^{3}\right),\left(a^{3}, b^{4}\right),\left(a^{2}, b^{2}\right)\right)\left(\left(a^{3}, b\right),\left(a^{2}, b^{2}\right),\left(a^{3}, b^{4}\right),\left(a^{2}, b^{3}\right)\right) \\
& \left(\left(a^{4}, b\right),\left(a, b^{3}\right),\left(a^{4}, b^{4}\right),\left(a, b^{2}\right)\right)\left(\left(a^{4}, b\right),\left(a, b^{2}\right),\left(a^{4}, b^{4}\right),\left(a, b^{3}\right)\right) \\
& \left((e, b),\left(e, b^{3}\right),\left(e, b^{4}\right),\left(e, b^{2}\right)\right)\left((e, b),\left(e, b^{2}\right),\left(e, b^{4}\right),\left(e, b^{3}\right)\right) \\
& \left((a, e),\left(a^{4}, e\right)\right)\left(\left(a^{2}, e\right),\left(a^{3}, e\right)\right)\left((a, e),\left(a^{4}, e\right)\right)\left(\left(a^{2}, e\right),\left(a^{3}, e\right)\right) \\
& (19) \\
& \left(a a^{5} a^{4} a^{18} a^{14} a^{15}\right)\left(a^{2} a^{7} a^{5} a^{17} a^{12} a^{14}\right)\left(a^{3} a^{9} a^{6} a^{16} a^{10} a^{13}\right) \\
& \left(a^{9} a a^{11} a^{10} a^{18} a^{8}\right)\left(a^{8} a^{2} a^{13} a^{11} a^{17} a^{6}\right)\left(a^{7} a^{3} a^{15} a^{12} a^{16} a^{4}\right)
\end{aligned}
$$

is a (6.2) complete mapping of the cyclic group $C_{19}$.

$$
\begin{gathered}
\left(a^{7} a^{14}\right)\left(a^{7} a^{14}\right)\left(a^{2} a^{10} a^{8} a^{19} a^{11} a^{13}\right)\left(a^{4} a^{9} a^{5} a^{17} a^{12} a^{16}\right)\left(a^{6} a a^{16} a^{15} a^{20} a^{5}\right) \\
\left(a^{9} a^{3} a^{15} a^{12} a^{18} a^{6}\right)\left(a a^{4} a^{3} a^{20} a^{17} a^{18}\right)\left(a^{10} a^{2} a^{13} a^{11} a^{19} a^{8}\right)
\end{gathered}
$$

is a $(K, 2)$ complete mapping of the cyclic group $C_{21}$ with $K=$ $\{2,2,6,6,6,6,6,6\}$.

(21)

$$
\begin{gathered}
{\left[\text { e } a^{7}\right]\left[\text { e } a^{7}\right]\left(a a^{4} a^{3} a^{13} a^{10} a^{11}\right)\left(a^{6} a a^{9} a^{8} a^{13} a^{5}\right)} \\
\left(a^{2} a^{6} a^{4} a^{12} a^{8} a^{10}\right)\left(a^{5} a^{2} a^{11} a^{9} a^{12} a^{3}\right)
\end{gathered}
$$


is a $(K, 2)$ near complete mapping of the cyclic group $C_{14}$ with $K=$ $\{2,2 ; 6,6,6,6\}$.

(22)

$$
\begin{aligned}
& \left(a a^{6} a^{5} a^{24} a^{19} a^{20}\right)\left(a^{2} a^{8} a^{6} a^{23} a^{17} a^{19}\right)\left(a^{4} a^{12} a^{8} a^{21} a^{13} a^{17}\right) \\
& \left(a^{12} a a^{14} a^{13} a^{24} a^{11}\right)\left(a^{11} a^{2} a^{16} a^{14} a^{23} a^{9}\right)\left(a^{9} a^{4} a^{20} a^{16} a^{21} a^{5}\right) \\
& \left(a^{3} a^{10} a^{7} a^{22} a^{15} a^{18}\right)\left(a^{10} a^{3} a^{18} a^{15} a^{22} a^{7}\right)
\end{aligned}
$$

is a $(6,2)$ complete mapping of the cyclic group $C_{25}$.

$$
\begin{gathered}
{\left[e b a^{3}\right]\left[e b a^{4}\right]\left(a^{2} a a^{4}\right)\left(a^{2} a a^{4}\right)\left(a^{3} b a b a^{5}\right)\left(a^{3} b a^{2} b a^{6}\right)\left(a^{6} b a^{6} b\right)} \\
\left(a^{6} b b a\right)\left(a^{5} b a^{2} b a^{4}\right)\left(a^{5} b a^{3} b a^{5}\right)
\end{gathered}
$$

is a $(3,2)$ near complete mapping of the dihedral group $D_{7}$.

(24) Since 2 is a primitive root of 13, the following sequence provides an $R_{11}$-sequencing of the cyclic group $C_{13}$ when written additively. (See [12] for a proof.)

$$
\sigma=(124836121195107)
$$

We find that

$$
\begin{aligned}
& \sigma^{2}=(14312910)(2861157) \\
& \sigma^{3}=\left(\begin{array}{lll}
1 & 8 & 12
\end{array}\right)(231110)(4697) \\
& \sigma^{4}=\left(\begin{array}{lll}
1 & 3 & 9
\end{array}(265)(41210)(8117)\right. \\
& \sigma^{6}=(112)(211)(49)(85)(310)(67) \text {. }
\end{aligned}
$$

When these five mappings are adjoined they define a $(K, 5)$ tight generalized complete mapping of the cyclic group $C_{13}$, where $K=$ $\{12,6,6,4,4,4,3,3,3,3,2,2, \ldots, 2\}$.

(25) Since a root $\alpha$ of the equation $\alpha^{2}=\alpha+1$ is a primitive element of the Galois field GF[9], the following sequence provides an $R_{7}$-sequencing of the additive group of the field.

$$
\sigma=\left(\begin{array}{llllllll}
1 & \alpha & \alpha+1 & -\alpha+1 & -1 & -\alpha & -\alpha-1 & \alpha-1
\end{array}\right)
$$

We find that

$$
\begin{aligned}
& \sigma^{2}=\left(\begin{array}{lllll}
1 & \alpha+1 & -1 & -\alpha-1
\end{array}\right)\left(\begin{array}{llll}
\alpha & -\alpha+1 & -\alpha & \alpha-1
\end{array}\right) \\
& \sigma^{4}=\left(\begin{array}{llll}
1 & -1
\end{array}\right)\left(\begin{array}{llll}
\alpha+1 & -\alpha-1
\end{array}\right)\left(\begin{array}{lll}
\alpha & -\alpha
\end{array}\right)\left(\begin{array}{lll}
-\alpha+1 & \alpha-1
\end{array}\right) .
\end{aligned}
$$


When these three mappings are adjoined they define a $(K, 3)$ tight generalized complete mapping of the group $G=\operatorname{gp}\left\{a: a^{3}=e\right\} \times$ $\operatorname{gp}\left\{b: b^{3}=e\right\}$ which we can write in the form

$$
\begin{aligned}
& \left(a b a b a b^{2} a^{2} b^{2} a^{2} b^{2} a^{2} b\right)\left(a a b a^{2} a^{2} b^{2}\right) \\
& \quad\left(b a b^{2} b^{2} a^{2} b\right)\left(a a^{2}\right)\left(a b a^{2} b^{2}\right)\left(b b^{2}\right)\left(a b^{2} a^{2} b\right) .
\end{aligned}
$$

(26) $\left(a a^{4} a^{3} a^{12} a^{9} a^{10}\right)\left(a^{2} a^{8} a^{6} a^{11} a^{5} a^{7}\right)$ is a strong $(6,1)$ complete mapping of the cyclic group $C_{13}$ which satisfies the additional condition required by Theorem 6.3 .

(27) ( $\left.a a b a^{2} a^{2} b^{2}\right)\left(b a b^{2} b^{2} a^{2} b\right)$ is a strong $(4,1)$ complete mapping of the group $C_{3} \times C_{3} \cong \operatorname{gp}\left\{a: a^{3}=e\right\} \times \operatorname{gp}\left\{b: b^{3}=e\right\}$.

(28) $\left(a a^{4} a^{3} a^{12} a^{9} a^{10}\right)\left(a^{2} a^{6} a^{4} a^{11} a^{7} a^{9}\right)\left(a^{5} a^{2} a^{10} a^{8} a^{11} a^{3}\right)\left(a^{6} a a^{8}\right.$ $\left.a^{7} a^{12} a^{5}\right)$ is a strong tight $(6,2)$ complete mapping of the cyclic group $C_{13}$ which satisfies the additional condition required by Theorem 6.3 .

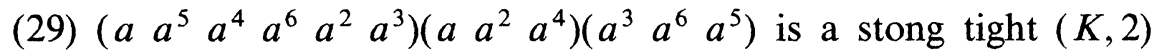
complete mapping of the cyclic group $C_{7}$ constructed by the methods of Theorem 6.2, where $K=\{6,3,3\}$.

(30) $\left[e b a^{3}\right]\left(a^{2} a a^{4}\right)\left(a^{3} b a b a^{5}\right)\left(a^{6} b a^{6} b\right)\left(a^{5} b a^{2} b a^{4}\right)$ is a strong $(K, 1)$ near complete mapping of the dihedral group $D_{7}=\operatorname{gp}\left\{a, b: a^{7}=\right.$ $\left.b^{2}=e, a b=b a^{-1}\right\}$, where $K=\{2 ; 3,3,3,3\}$.

\section{REFERENCES}

[1] R. A. Bailey, Quasi-complete latin squares: construction and randomization, J. Royal Statist. Soc., Series B, 46 (1984), 323-334.

[2] F. E. Bennett, E. Mendelsohn and N. S. Mendelsohn, Resolvable perfect cyclic designs, J. Combinatorial Theory (A), 29 (1980), 142-150.

[3] F. E. Bennett and D. Sotteau, Almost resolvable decompositions of $K_{n}^{*}$, J. Combinatorial Theory (B), 30 (1981), 228-232.

[4] J. C. Bermond, A. Germa and D. Sotteau, Resolvable decompositions of $K_{n}^{*}$, J. Combinatorial Theory (A), 26 (1979), 179-185.

[5] C. J. Colbourn and M. J. Colbourn, Disjoint cyclic Mendelsohn triple systems, Ars Combinatoria, to appear.

[6] J. Dénes and A. D. Keedwell, Latin Squares and their Applications, (Akadémiai Kiadó, Budapest/English Universities Press, London/Academic Press, New York, 1974.)

[7] R. J. Friedlander, B. Gordon and P. Tannenbaum, Partitions of groups and complete mappings, Pacific J. Math., 92 (1981), 283-293.

[8] A. Gewirtz and D. F. Hsu, On neofield graphs, to appear.

[9] D. F. Hsu, Cyclic Neofields and Combinatorial Designs, Springer-Verlag, 1980, Lecture Notes in Mathematics, No. 824. 
[10] D. F. Hsu and A. D. Keedwell, Generalized complete mappings, neofields, sequencings and block designs. I, Pacific J. Math., 111 (1984), 317-332.

[11] A. D. Keedwell, On orthogonal latin squares and a class of neofields, Rend. Mat., (Roma) (5) 25 (1966), 519-561.

[12] On $R$-sequenceability and $R_{h}$-sequenceability of groups, Annals of Discrete Math., 18 (1983), 535-548.

[13] N. S. Mendelsohn, Perfect cyclic designs, Discrete Math., 20 (1977), 63-68.

Received February 11, 1983 and in revised form November 2, 1983. The work of the first author was supported in part by Fordham University Faculty Research Grant \#071225.

UNIVERSITY OF SURREY

GUILDFORD, SURREY, GU2 5XH

ENGLAND 


\section{PACIFIC JOURNAL OF MATHEMATICS EDITORS}

DONALD BABBITT (Managing Editor)
University of California
Los Angeles, CA 90024
CHARLES R. DEPrIMA
California Institute of Technology
Pasadena, CA 91125
R. FINN
Stanford University
Stanford, CA 94305

\author{
HERMANN FLASCHKa \\ University of Arizona \\ Tucson, AZ 85721 \\ RAMESH A. GANGOLLI \\ University of Washington \\ Seattle, WA 98195 \\ ROBION KIRBY \\ University of California \\ Berkeley, CA 94720 \\ C. C. Moore \\ University of California \\ Berkeley, CA 94720
}

Hugo Rossi

University of Utah

Salt Lake City, UT 84112

H. SAMELSON
Stanford University
Stanford, CA 94305

HAROLD STARK

University of California, San Diego

La Jolla, CA 92093

\section{ASSOCIATE EDITORS}
R. ARens
E. F. BECKENBACH (1906-1982)
B. H. NeUmanN
F. WOLF
K. YOSHIDA

\begin{tabular}{ll}
\multicolumn{2}{c}{ SUPPORTING INSTITUTIONS } \\
UNIVERSITY OF ARIZONA & UNIVERSITY OF OREGON \\
UNIVERSITY OF BRITISH COLUMBIA & UNIVERSITY OF SOUTHERN CALIFORNIA \\
CALIFORNIA INSTITUTE OF TECHNOLOGY & STANFORD UNIVERSITY \\
UNIVERSITY OF CALIFORNIA & UNIVERSITY OF HAWAII \\
MONTANA STATE UNIVERSITY & UNIVERSITY OF TOKYO \\
UNIVERSITY OF NEVADA, RENO & UNIVERSITY OF UTAH \\
NEW MEXICO STATE UNIVERSITY & WASHINGTON STATE UNIVERSITY \\
OREGON STATE UNIVERSITY & UNIVERSITY OF WASHINGTON
\end{tabular}

The Supporting Institutions listed above contribute to the cost of publication of this Journal, but they are not owners or publishers and have no responsibility for its content or policies.

\footnotetext{
Mathematical papers intended for publication in the Pacıfic Journal of Mathematıcs should be in typed form or offset-reproduced (not dittoed), double spaced with large margins. Please do not use built up fractions in the text of the manuscript. However, you may use them in the displayed equations. Underline Greek letters in red, German in green, and script in blue. The first paragraph must be capable of being used separately as a synopsis of the entire paper. In particular it should contain no bibliographic references. Please propose a heading for the odd numbered pages of less than 35 characters. Manuscripts, in triplicate, may be sent to any one of the editors. Please classify according to the scheme of Math. Rev ews, Index to Vol. 39. Supply name and address of author to whom proofs should be sent. All other communications should be addressed to the managing editor, or Elaine Barth, University of California, Los Angeles, California 90024.

There are page-charges associated with articles appearing in the Pacific Journal of Mathematics. These charges are expected to be paid by the author's University, Government Agency or Company. If the author or authors do not have access to such Institutional support these charges are waived. Single authors will receive 50 free reprints; joint authors will receive a total of 100 free reprints. Additional copies may be obtained at cost in multiples of 50 .
}

The Pacific Journal of Mathematics is issued monthly as of January 1966. Regular subscription rate: $\$ 190.00$ a year (5 Vols., 10 issues). Special rate: $\$ 66.00$ a year to individual members of supporting institutions.

Subscriptions, orders for numbers issued in the last three calendar years, and changes of address should be sent to Pacific Journal of Mathematics, P.O. Box 969, Carmel Valley, CA 93924, U.S.A. Old back numbers obtainable from Kraus Periodicals Co., Route 100, Millwood, NY 10546.

The Pacific Journal of Mathematics at P.O. Box 969, Carmel Valley, CA 93924 (ISSN 0030-8730) publishes 5 volumes per year. Application to mail at Second-class postage rates is pending at Carmel Valley, California, and additional mailing offices. Postmaster: Send address changes to Pacific Journal of Mathematics, P.O. Box 969, Carmel Valley, CA 93924.

PUBLISHED BY PACIFIC JOURNAL OF MATHEMATICS, A NON-PROFIT CORPORATION Copyright $\odot 1985$ by Pacific Journal of Mathematics 


\section{Pacific Journal of Mathematics}

\section{Vol. 117, No. 2 \\ February, 1985}

Robert Walter Bagley, Ta-Sun Wu and J. S. Yang, On a class of topological groups more general than SIN groups ............... 209

Bruce Alan Barnes, Algebraic elements of a Banach algebra modulo an ideal ...............................................219

Howard D. Fegan and Peter Gilkey, Invariants of the heat equation .......233

Erica Flapan, Necessary and sufficient conditions for certain homology

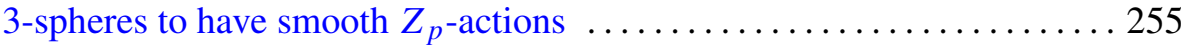

Philip R. Heath, Product formulae for Nielsen numbers of fibre maps . . . . 267

Derbiau Frank Hsu and A. Donald Keedwell, Generalized complete mappings, neofields, sequenceable groups and block designs. II ...... 291

Taqdir Husain, Orthogonal primitive idempotents and Banach algebras

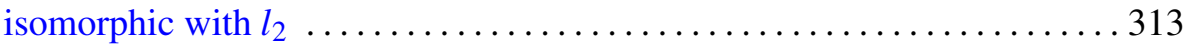

Gary M. Lieberman, Regularized distance and its applications . . . . . . . 329

William W. Menasco, Determining incompressibility of surfaces in alternating knot and link complements ............................ 353

Benjamin Muckenhoupt, Weighted reverse weak type inequalities for the Hardy-Littlewood maximal function $\ldots \ldots \ldots \ldots \ldots \ldots \ldots \ldots \ldots \ldots \ldots \ldots$

John Dacey O'Neill, Direct summands of direct products of slender

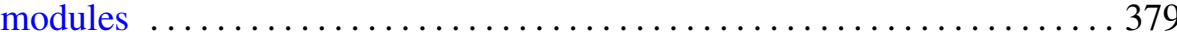

Andreas Rapp, Elimination of Malitz quantifiers in stable theories 387

Francisco José Ruiz, A unified approach to Carleson measures and $A_{p}$ weights

Hanamatagouda Pandappa Sankappanavar, Heyting algebras with dual

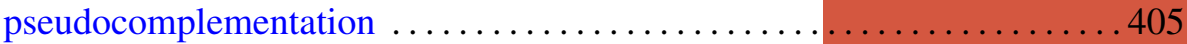

Annette Sinclair, Strong Carleman and strong uniform approximation . . . . 417

Masaaki Suzuki, The generalized Schwarz lemma for the Bergman metric

Brian Thorpe and Ludwig Tomm, Universal approximation by regular

weighted means 